Supplementary Materials

\title{
An all-ceramic, anisotropic, and flexible aerogel insulation material
}

Lu An ${ }^{\dagger}$, Jieyu Wang \#, Donald Petit ${ }^{\dagger}$, Jason N. Armstrong ${ }^{\dagger}$, Karen Hanson ${ }^{\natural}$, Jason

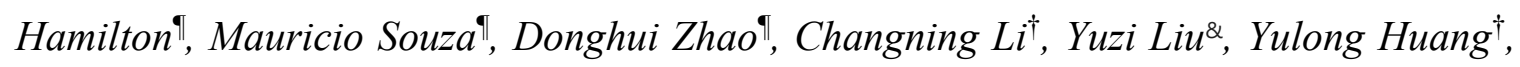
Yong $\mathrm{Hu}^{\dagger}$, Zheng $\mathrm{Li}^{\dagger, \dagger}$, Zefan Shao ${ }^{\dagger}$, André Omer Desjarlais ${ }^{\prime}$, and Shenqiang Ren ${ }^{\dagger, \S, \#^{*}}$

${ }^{\dagger}$ Department of Mechanical and Aerospace Engineering, University at Buffalo, The State University of New York, Buffalo, NY 14260, USA

\# Department of Chemistry, University at Buffalo, The State University of New York, Buffalo, NY 14260, USA

"Unifrax Inc., Tonawanda, NY 14150, USA

\& Center for Nanoscale Materials, Argonne National Laboratory, IL 60439

$\$$ College of Material Science and Technology, Nanjing University of Aeronautics and Astronautics, Nanjing, 210016, China

\| Building Envelopes Program, Oak Ridge National Laboratory, Oak Ridge, TN 37830, USA

${ }^{\S}$ Research and Education in Energy, Environment \& Water (RENEW), University at Buffalo, The State University of New York, Buffalo, NY 14260, USA

Email: shenren@buffalo.edu 
Table 1 Chemical composition of aluminoborosilicate fibers

\begin{tabular}{lllllll}
\hline & $\mathrm{SiO}_{2}$ & $\mathrm{Al}_{2} \mathrm{O}_{3}$ & $\mathrm{~B}_{2} \mathrm{O}_{3}$ & $\mathbf{C a O}$ & $\mathrm{MgO}$ & Others \\
\hline wt. $\%$ & $50 \sim 56$ & $13 \sim 16$ & $5.8 \sim 10$ & $15 \sim 24$ & $<5.5$ & $<3.43$ \\
\hline
\end{tabular}

a
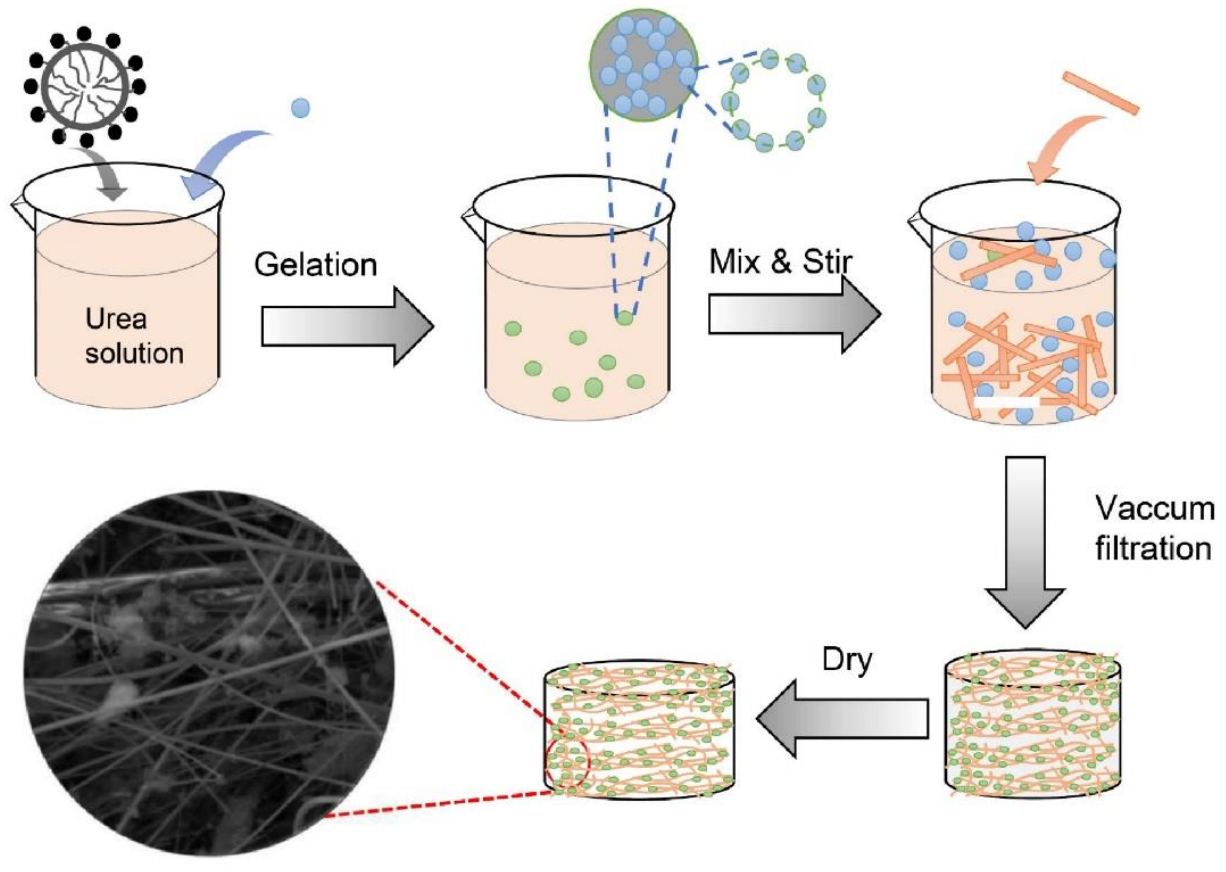

$\mathrm{b}$

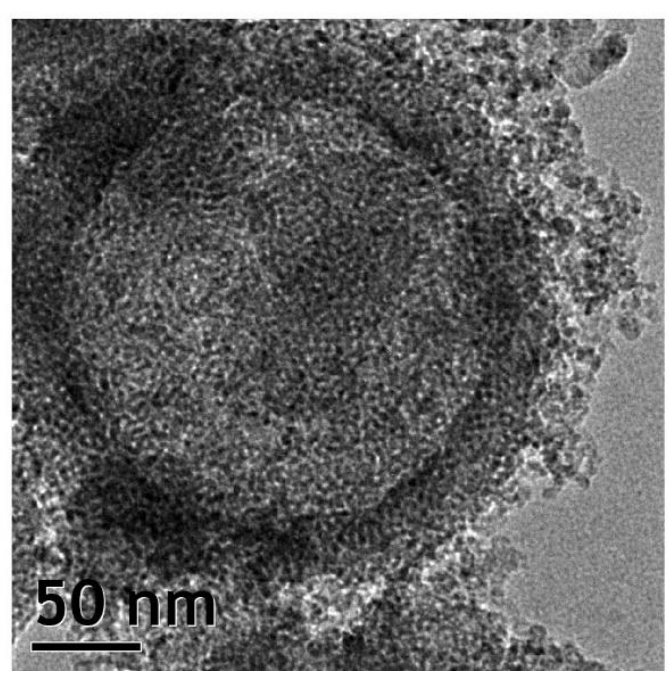

Figure S1. (a) Schematic illustrations of the synthesis of P-FRAero through silica precursor approach. (b) Nano-porous silica aerogel shell. 
a
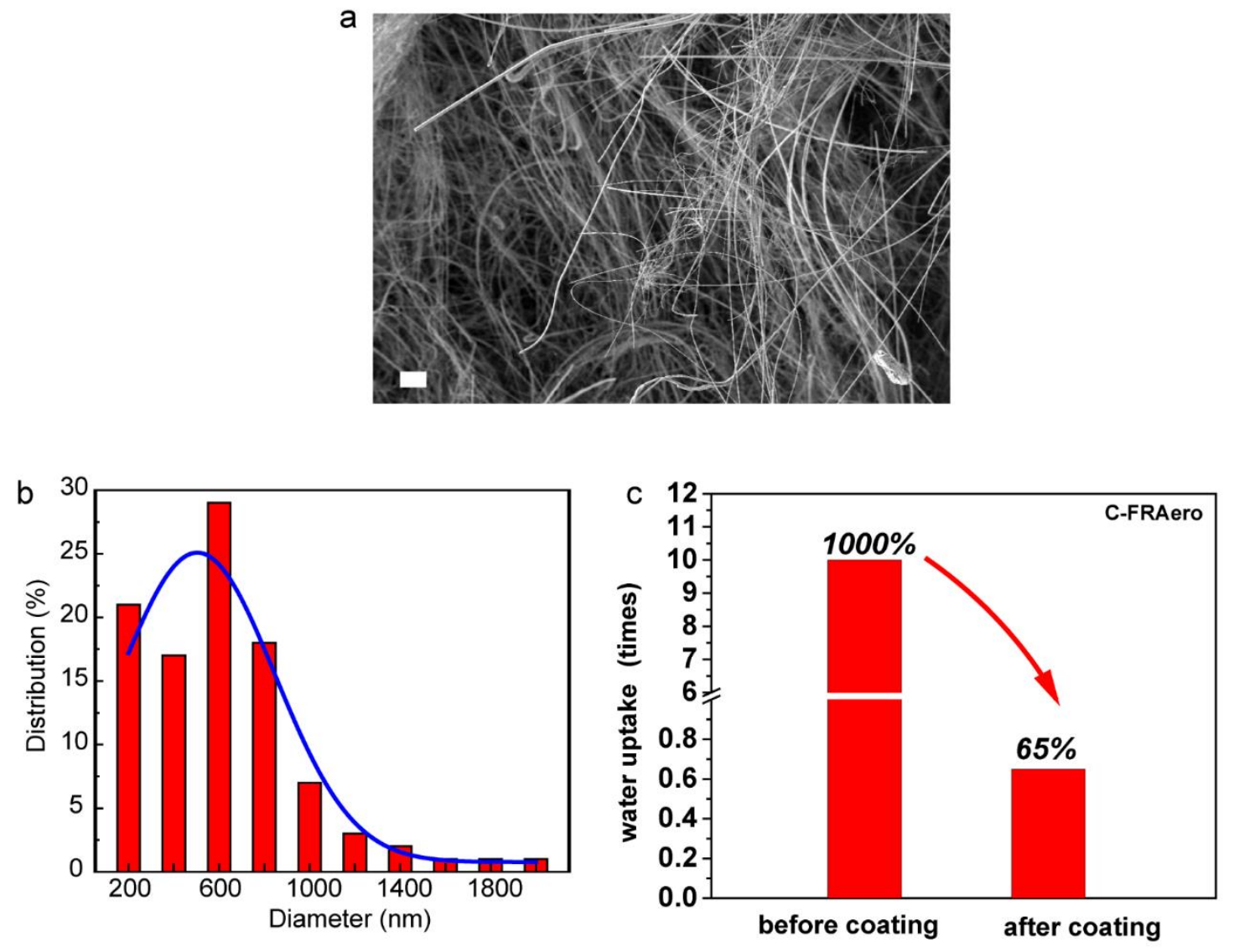

Figure S2. (a) SEM image of aluminoborosilicate fiber. (b) The diameter distribution of aluminoborosilicate fiber. (c) Water uptake of C-FRAero before and after coating. 


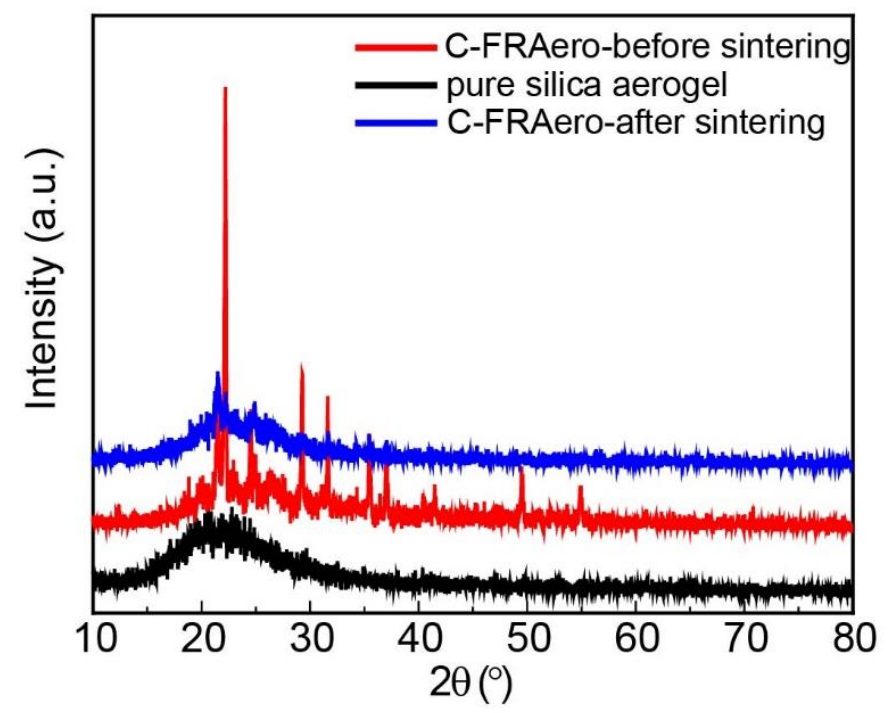

Figure S3. XRD patterns of silica aerogel, fiber-aerogel mat with and without sintering. The C-FRAero without sintering show the sharp XRD peaks, corresponding to that of Urea. After $400^{\circ} \mathrm{C}$ sintering treatment, the C-FRAero shows the broad peak around $2 \theta$ equaling to 20 degree, which indicates amorphous structure of fibers and silica aerogel. 
a

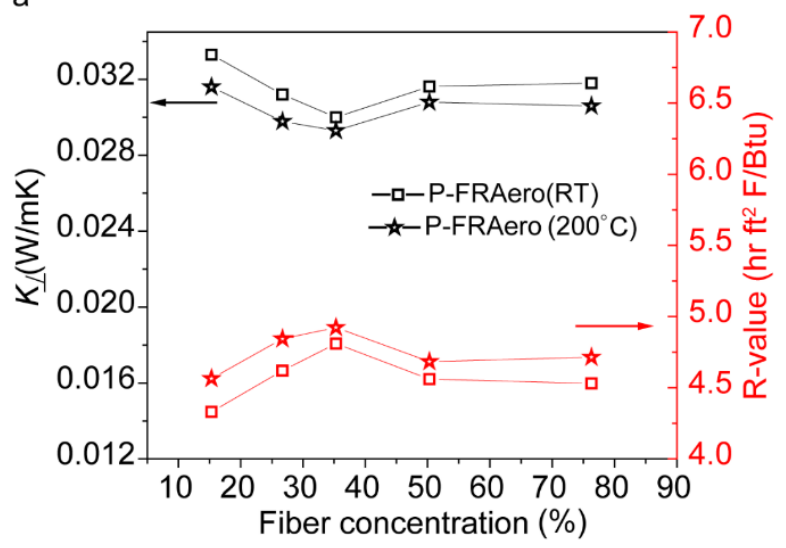

b

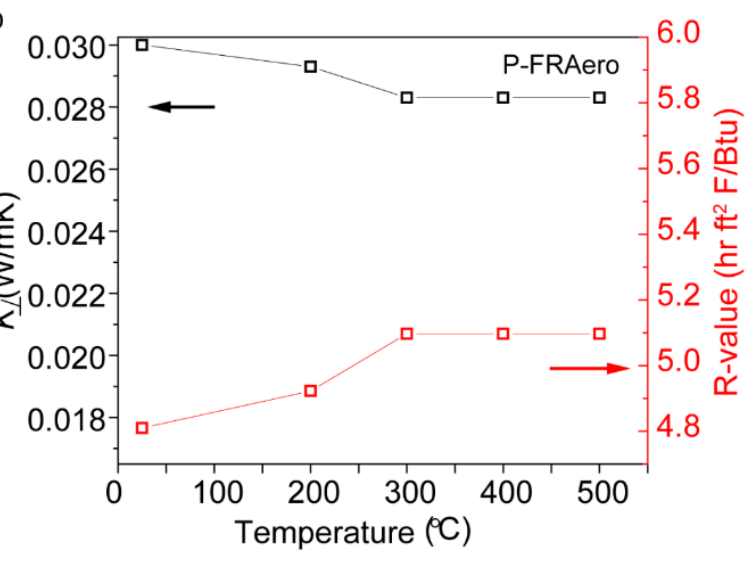

Figure S4. Thermal properties of P-FRAero. (a) Out of plane direction thermal conductivity $K_{\perp}$ vs. fiber concentration of C- FRAero sheets before and after $200{ }^{\circ} \mathrm{C}$ sintering. (b) $K_{\perp}$ vs. sintering temperature T for 35 wt.\% P- FRAero paper sheet. 

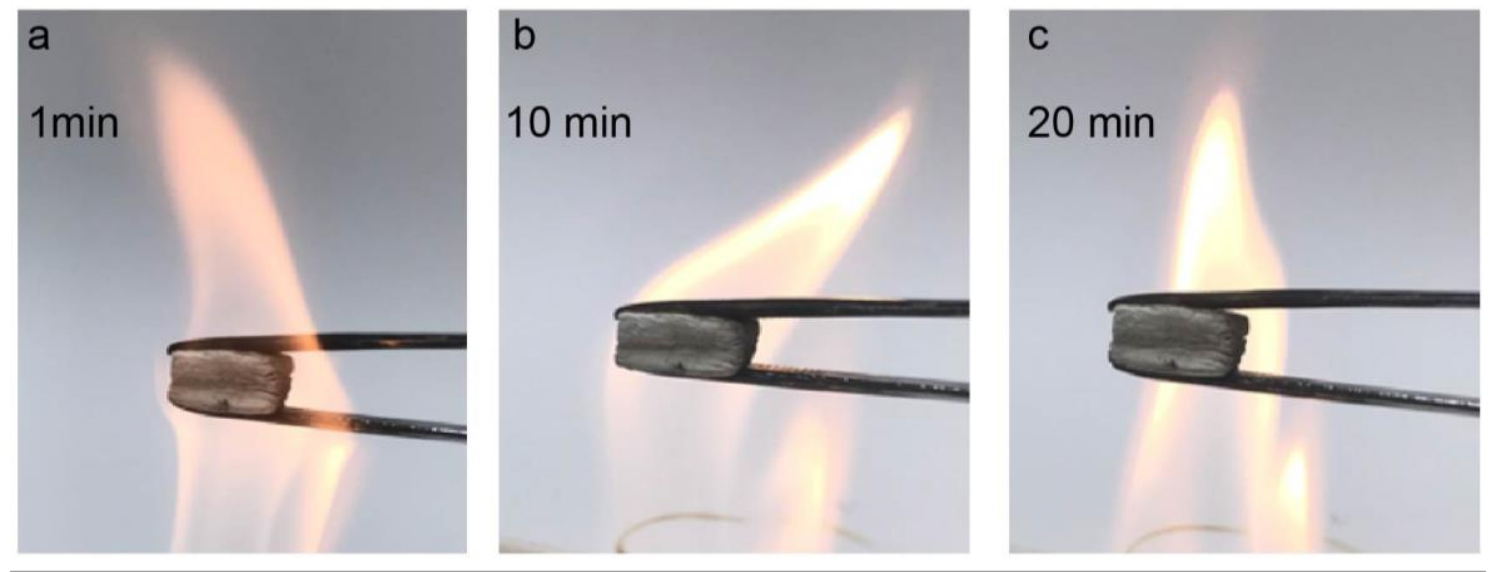

Figure S5. Fire retardant performance of C-FRAero paper sheet in the flame for $20 \mathrm{~min}$. 


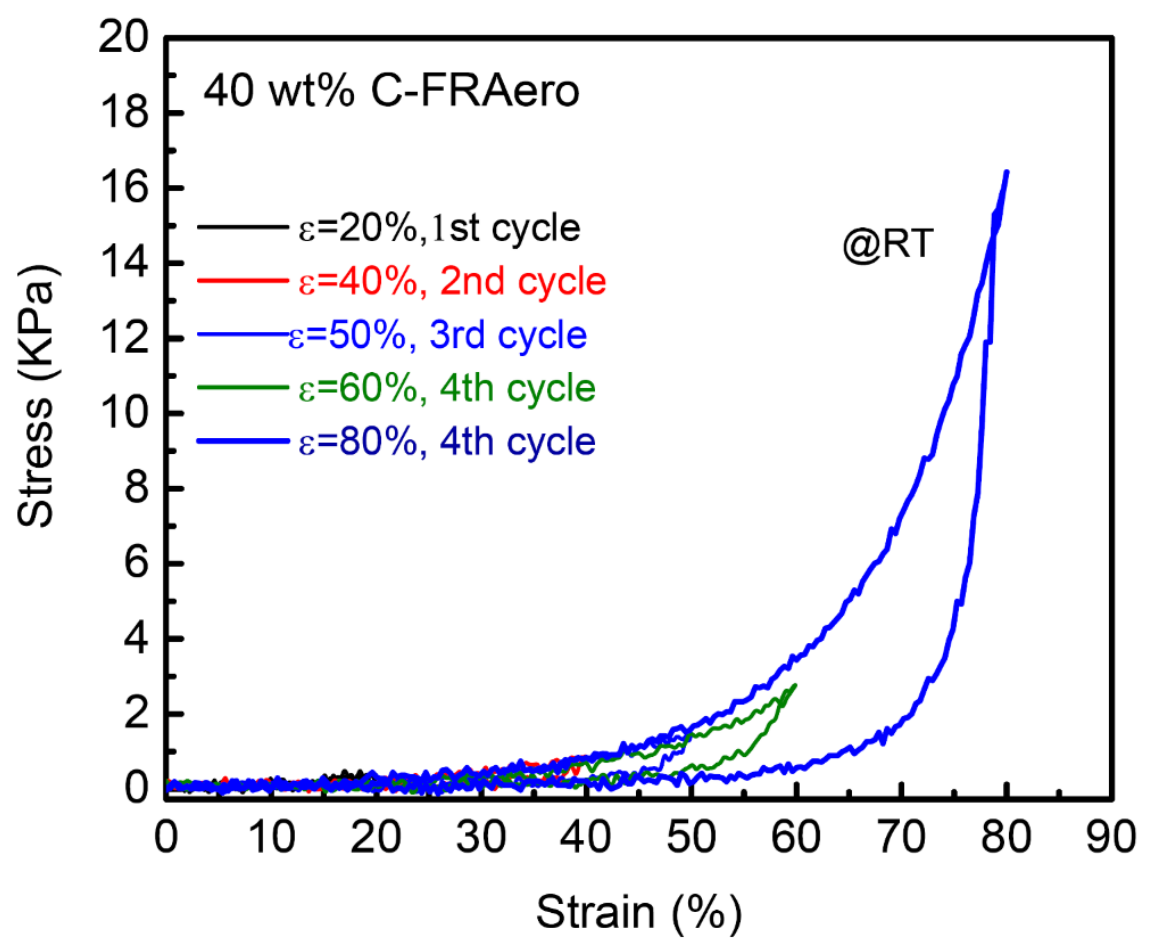

Figure S6. Multiple compressive stress-strain curves of $40 \mathrm{wt}$ \% C-FRAero paper sheet under room temperature. 


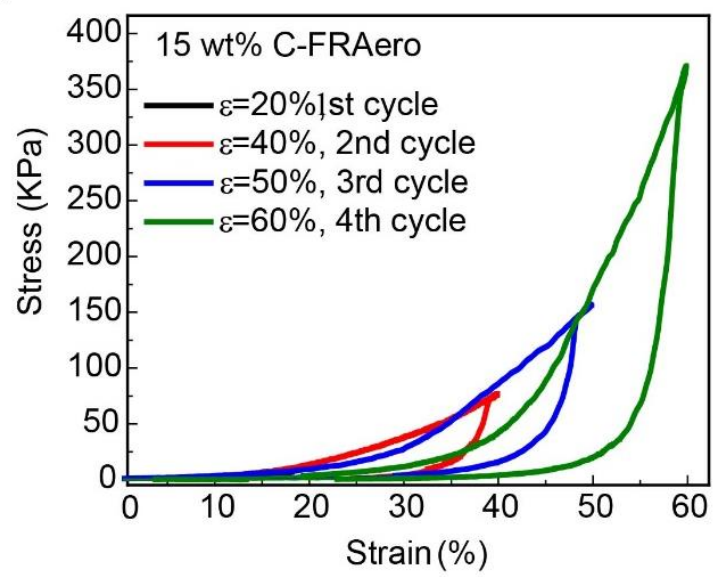

b

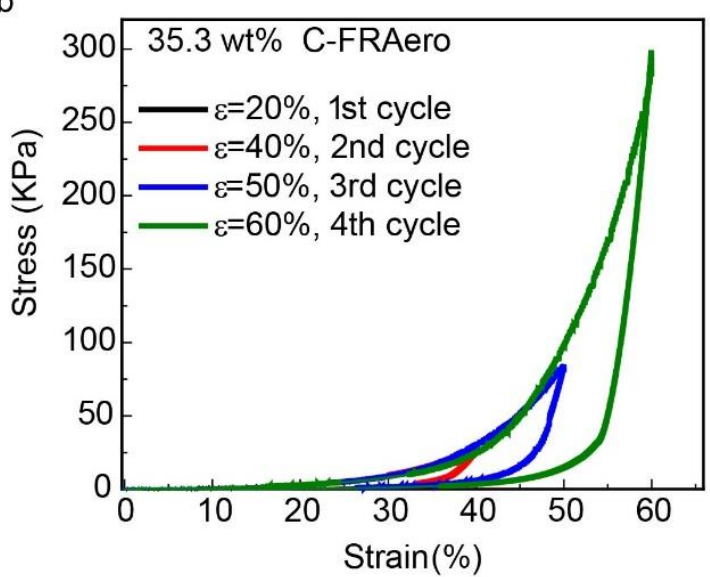

Figure S7. Multiple compressive stress-strain curves of (a) 15 wt.\% C-FRAero paper sheet, (b) 35.3 wt.\% C-FRAero paper sheet after $400^{\circ} \mathrm{C}$ sintering. 


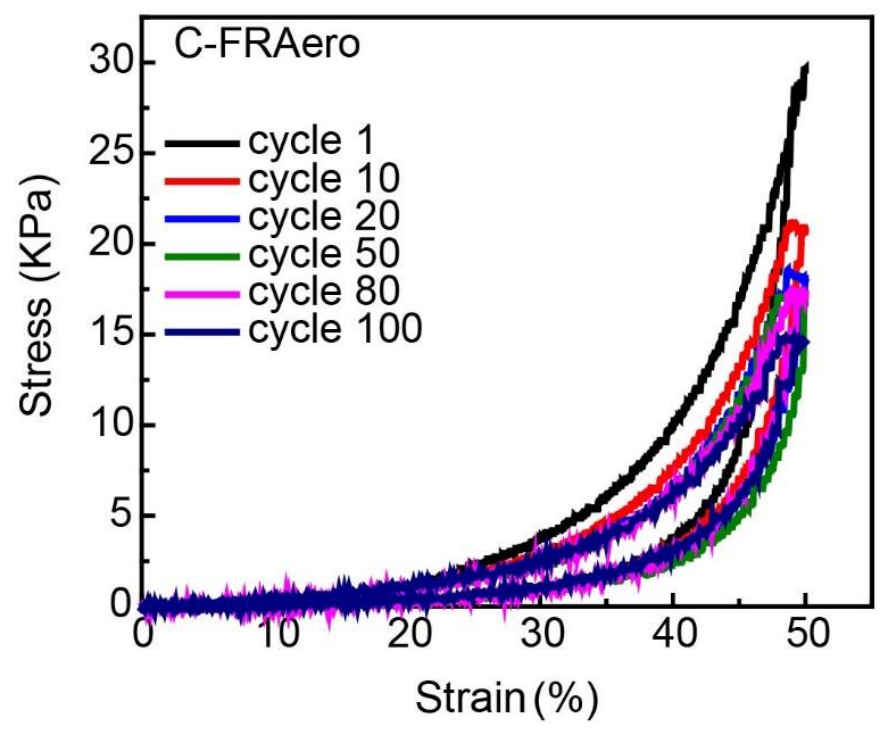

Figure S8. 100-cycle of compressive stress-strain curves of $40 \mathrm{wt}$ \% $\%$ C-FRAero paper sheet. 


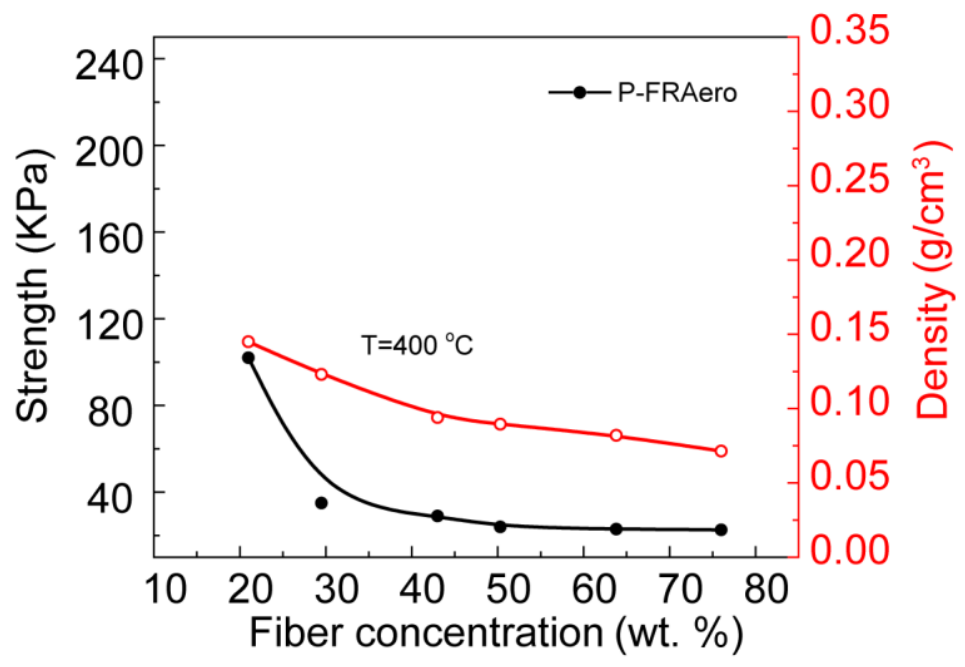

Figure S9. Out-of-plane direction compressive strength and density vs. fiber concentration for P-FRAero sheets. 